POLYMERS

\title{
Randomness takes control
}

4

\section{Other} monomers could be explored with the likelihood of fully recapitulating the properties of proteins
Proton transport across biological membranes is controlled in part by the structure and behaviour of proteins within the membranes. Making artificial channels with such specific structures and chemical diversity is challenging and, as yet, there are no synthetic systems with the same transport performance as biological membranes.

Now, writing in Nature, Ting Xu and colleagues report single-chain heteropolymers that enable the transport of protons across lipid bilayers at a similar rate to transport across biological membranes. In the presence of alkali metal cations and protons, this transport is selective towards protons.

When considering the whole chain, the arrangement of monomers in the heteropolymers is statistically random. However,



Credit: Reproduced from Jiang, T. et al (2020), Springer Nature Limited these random heteropolymers can also be considered as comprising segments with different cumulative hydrophobicities. "Thus, it is feasible to hypothetically break the chain into building blocks like lego pieces," says $\mathrm{Xu}$. "These lego pieces can be stitched together in a non-specific way to release the sequence specificity requirements to recapitulate protein behaviour."

The probability of realizing these lego pieces depends on the controlled randomness that is inherent to the design and synthesis of the random heteropolymer. "Thus, the system can exhibit rather defined behaviour albeit of sequence randomness at the monomeric level," says Xu.

Three different types of segments make up the random heteropolymer: they prefer either water, the lipid bilayer or the interface. $\mathrm{Xu}$ and colleagues find that a four-monomerbased polymer is needed for the chemical diversity within the polymer to be sufficient that all these behaviours are realised and for transmembrane proton transport to occur. More specifically, the inclusion of two hydrophobic monomers ensures that the heteropolymer can insert into the lipid bilayer, and a hydrophilic monomer balances the overall hydrophilicity. A fourth monomer reduces the likelihood of aggregation of the polymer.

Using the techniques of fluorescence microscopy and differential scanning calorimetry, $\mathrm{Xu}$ and colleagues reveal that the random heteropolymers insert into lipid bilayers, rather than reside on the surface. The distribution of the random heteropolymer within the lipid bilayer was further investigated using molecular dynamics simulations. From the simulations, the hydrophobic segments in the bilayer are seen to be fairly static whereas the hydrophilic segments in the water move. The hydrophilic segments in the bilayer move more slowly and provide hydrogen-bonded chains which are vital for proton transport. "Our results show the importance of the combination of both static and dynamic segments in the designed membrane," says Xu.

"The monomers chosen in this example are by no means specific," says Xu. "Other monomers could be explored with the likelihood of fully recapitulating the properties of proteins."

The researchers aim to identify the design rules to create polymers that are truly protein-like. This example reveals the ability of a synthetic polymer to mimic transmembrane proteins. "The longterm goal is to see how far we can go - for example, the mimicking of structural proteins, virus capsids and enzymes, as well as controlling oligomerization in proteins that can lead to responsive behaviour," says $\mathrm{Xu}$.

Alison Stoddart ORIGINAL ARTICLE Jiang, T. et al. Single-chain heteropolymers transport protons selectively and rapidly. Nature 577, 216-220 (2020) 